\title{
Comparison of the effects of coenzyme Q10 and Centrum multivitamins on semen parameters, oxidative stress markers, and sperm DNA fragmentation in infertile men with idiopathic oligoasthenospermia
}

\author{
Ahmed T Alahmar ${ }^{1}$, Rajender Singh ${ }^{2}$ \\ 'Department of Medical Physiology, College of Medicine, University of Babylon, Hillah, Iraq; ${ }^{2}$ CSIR-Central Drug Research Institute, Lucknow, India
}

Objective: Oxidative stress and sperm DNA fragmentation (SDF) have been linked to idiopathic male infertility (IMI). Various antioxidants have been tried to improve semen parameters and fertility potential in IMI patients, but with inconsistent results. The study aimed to compare the effects of coenzyme Q10 (CoQ10) and Centrum multivitamins on semen parameters, seminal antioxidant capacity, and SDF in infertile men with idiopathic oligoasthenospermia (OA).

Methods: This prospective controlled clinical study involved 130 patients with idiopathic OA and 58 fertile controls. The patients were divided randomly into two groups: the first group received CoQ10 (200 mg/day orally) and the second group received Centrum multivitamins (1 tablet/day) for 3 months. Semen parameters, CoQ10 levels, reactive oxygen species (ROS), total antioxidant capacity (TAC), catalase, SDF, and serum hormone levels (follicle-stimulating hormone, luteinizing hormone, testosterone, and prolactin) were compared at baseline and after 3 months.

Results: Both CoQ10 and Centrum improved sperm concentration and motility, but the improvement was greater with Centrum therapy $(p<0.05)$. Similarly, both therapies improved antioxidant capacity, but TAC and catalase improvement was greater $(p<0.01$ and $p<0.001$ respectively) with CoQ10, whereas ROS $(p<0.01)$ and SDF $(p<0.001)$ improvements were greater with Centrum administration. Centrum therapy was associated with reduced serum testosterone $(p<0.05)$.

Conclusion: In conclusion, both CoQ10 and Centrum were effective in improving semen parameters, antioxidant capacity, and SDF, but the improvement was greater with Centrum than with CoQ10. Therefore, Centrum - as a source of combined antioxidants - may provide more effective results than individual antioxidants such as CoQ10 in the treatment of infertile men with idiopathic OA.

Keywords: Centrum; Coenzyme Q10; Idiopathic oligoasthenozoospermia; Oxidative stress

Received: September 2, 2021 - Revised: November 8, 2021 · Accepted: December 1, 2021 Corresponding author: Ahmed T Alahmar

Department of Medical Physiology, College of Medicine, University of Babylon, Hillah, Iraq 51001, Iraq

Tel: +964-78-0818-0900 E-mail: ahmed.t.alahmar@gmail.com

This is an Open Access article distributed under the terms of the Creative Commons Attribution Non-Commercial License (http://creativecommons.org/licenses/by-nc/4.0/) which permits unrestricted non-commercial use, distribution, and reproduction in any medium, provided the original work is properly cited.

\section{Introduction}

Infertility is defined as the failure to achieve pregnancy after 12 months or more of regular unprotected intercourse [1]. Infertility is a global health issue affecting approximately 48 million couples worldwide [2], and male factors are considered to be the contributor in approximately $50 \%$ of infertile couples [3]. The etiology of male infertility is complex and includes underlying factors such as cryptorchidism, varicocele, genital tract infections, genetic mutations, immunological, endocrine disorders, systemic diseases, and environmental 
factors [4]. However, in 30\%-50\% of all male infertility cases, the underlying cause of semen abnormalities remains unknown, leading to their classification as idiopathic male infertility (IMI) [5]. Oxidative stress (OS) is also a contributing factor in a significant number of infertile men. OS is defined as the distortion of the prooxidant-antioxidant balance, resulting in elevated levels of reactive oxygen species (ROS) [6]. Increased levels of seminal ROS have been found in 30\%$80 \%$ of men with infertility [7]. Excessive ROS could be due to immature sperm, white blood cells, testicular torsion, cryptorchidism, varicocele, aging, infection, tumors, radiation, chemotherapy, environmental toxins, smoking, alcohol use, or systemic disease [8]. An increase in OS levels can lead to sperm plasma membrane damage due to lipid peroxidation, reduced sperm motility, reduced fertilization, and sperm DNA fragmentation (SDF) [9].

Spermatozoa possess limited-capacity antioxidant defense mechanisms that protect against ROS-induced damage [10]. Intrinsic protection is mediated by enzymatic antioxidants such as superoxide dismutase, catalase (CAT), glutathione peroxidase (GPx), and glutathione S-transferase, and non-enzymatic antioxidants including urate, carnitine, glutathione, coenzyme Q10 (CoQ10), and vitamins C and E [11]. A low total antioxidant capacity (TAC) in infertile men has been attributed to a significant reduction in both enzymic and non-enzymic antioxidants [12]. A reduction in the antioxidant defense and OS may also result in SDF, which has been associated with abnormal semen parameters, reduced fertilization, and conception rates, as well as increased rates of malignancies and neurological disorders in offspring [13].

CoQ10, which is a component of the mitochondrial respiratory chain, exerts antioxidant effects [14]. CoQ10 deficiency has been associated with lower sperm motility, count, and male infertility, while CoQ10 supplementation has been shown to improve sperm count and sperm morphology in infertile men [15]. We and others have reported that CoQ10 therapy increased antioxidant capacity in men with idiopathic oligoasthenospermia (OA) [16-18]. However, studies exploring the impacts of CoQ10 on seminal antioxidant capacity and SDF in men with idiopathic OA are limited. Other studies have tried combinations of multiple antioxidants to treat male infertility and have reported variable effects on semen parameters, antioxidant capacity, and SDF $[19,20]$. Furthermore, there is a lack of agreement on the type, dose, or the use of the individual or combined antioxidants in IMI [17]. Therefore, the present study aimed to compare the effects of CoQ10 and Centrum multivitamins (containing 26 vitamins and minerals) on semen parameters, OS markers, and SDF in infertile men with idiopathic OA.

\section{Methods}

This prospective clinical study was conducted with a 3-month follow-up period. One hundred and thirty patients with idiopathic $\mathrm{OA}$ and 58 fertile men as the control group were enrolled at the Fertility Clinic, Babyl, Iraq, from August 2018 to February 2019. A comprehensive fertility assessment was performed for all participants. Nine patients did not complete the study and were excluded. Patients were allocated randomly to one of two groups (each containing 65 patients). The first group received a daily dose of $200 \mathrm{mg}$ of CoQ10 (in the form of ubiquinol) (AMS, Woodinville, WA, USA) as a single oral dose for 3 months [21]. The second group received Centrum multivitamins (Pfizer, New York, NY, USA) as 1 tablet per day orally containing 26 vitamins and minerals for 3 months. Semen analysis findings, seminal CoQ10 levels, ROS, TAC, CAT, SDF, and serum hormone levels (follicle-stimulating hormone [FSH], luteinizing hormone [LH], testosterone, and prolactin) levels were compared at baseline and after 3 months. The primary endpoint was improvement in semen parameters, and the secondary endpoint was improvement in seminal antioxidant markers. The sample size was calculated using $80 \%$ power and a $5 \%$ level of error, yielding 54 for each group.

The study was approved by the Research Ethical Committee of the University of Sumer (EC/2018/8879). All the participants provided an informed consent before enrollment. All procedures followed were in accordance with the ethical standards of the responsible committee on human experimentation (institutional and national) and with the Helsinki Declaration of 1975, as revised in 2008.

\section{Eligibility criteria}

Fertile controls had a history of fathering a child in the last 2 years, with normal seminal fluid analysis findings and normal female fertility assessment. The patients included in the study had a history of infertility of 1 year or more without the use of contraception. OA was defined according to the World Health Organization (WHO) 2010 criteria [22]. Patients were excluded if they had genital infections, azoospermia, cryptorchidism, varicocele, testicular trauma or surgery, an endocrine disorder, systemic disease, relevant medications, smoking, alcohol, recent administration of antioxidants, and female factor infertility.

\section{Semen analysis}

Semen samples were collected by masturbation following a period of abstinence of 2-3 days. The semen sample was collected in a special container, followed by incubation at $37^{\circ} \mathrm{C}$ until semen liquefaction, and then semen analysis was performed within 1 hour following the WHO manual criteria (fifth edition, 2010) [22]. Two semen analyses were performed at baseline and after 3 months, and mean 
values were analyzed as the results of both analyses. All semen analysis tests were performed by the same investigator (ATA) to ensure data consistency.

\section{Measurements of seminal $\mathrm{CoQ} 10$ concentrations}

Seminal CoQ10 levels were measured using high-performance liquid chromatography (HPLC) using an ultraviolet (UV) detector at 275 $\mathrm{nm}$ and calculated using a published method [23]. Reverse-phase HPLC with UV detection using coenzyme Q9 as the internal standard was utilized to obtain seminal CoQ10 levels.

\section{Seminal ROS measurements}

Semen samples were centrifuged at 3,000 rpm for 5 minutes to obtain seminal plasma and then stored at $-20^{\circ} \mathrm{C}$. A manual method was used for ROS measurement as previously described by Venkatesh et al. [24]. To $400 \mu \mathrm{L}$ of liquefied neat semen, $10 \mu \mathrm{L}$ of luminol (5-amino-2,3,-dihydro-1,4-phthalazinedione; Sigma, St. Louis, MO, USA), prepared as $5 \mathrm{mM}$ stock in dimethyl sulfoxide (DMSO), was added. Furthermore, $10 \mu \mathrm{L}$ of $5 \mathrm{mM}$ luminol in DMSO served as the blank, and $25 \mu \mathrm{L}$ of $\mathrm{H}_{2} \mathrm{O}_{2}$ with $10 \mu \mathrm{L}$ of luminol was used as a positive control. The luminol-dependent chemiluminescence served as an indicator of ROS levels.

\section{Measurement of seminal TAC and CAT}

TAC was estimated with a colorimetric method using a total antioxidant capacity assay kit (\#E-BC-K136; Elabscience, Houston, TX, USA). Seminal plasma CAT activity was assessed using a CAT Assay Kit (\#E-BC-K031, Elabscience) using the protocol recommended by the manufacturer.

\section{Chromatin dispersion test}

Sperm chromatin dispersion was tested using the Halosperm kit (Halotech DNA, Madrid, Spain). The test principle is that sperm with SDF do not exhibit the halo of dispersed DNA loops that is observed in sperm without SDF, after acid denaturation and removal of nuclear proteins. The nucleoids from spermatozoa with SDF show no or minimal dispersion halo. Bright-field microscopy with Diff-Quik staining was utilized to examine the halos. SDF, defined as the ratio (expressed as a percentage) of sperm with SDF to total spermatozoa, was calculated using a previously published method [25].

\section{Hormonal assays}

Venipuncture was performed to collect blood samples $(5 \mathrm{~mL})$ using clean, plain labeled tubes. The samples were allowed to clot, and centrifugation was performed at 3,000 rpm for 10 minutes for analysis of hormones. Serum FSH, LH, testosterone, and prolactin levels were estimated using enzyme-linked fluorescent assays with the
Mini Vidas system (bioMérieux, Marcy l'Etoile, France).

\section{Statistical analysis}

IBM SPSS ver. 24 (IBM Corp., Armonk, NY, USA) was used for the data analysis. The results were expressed as mean \pm standard deviation. Data normality was assessed using Kolmogorov-Smirnov test. The paired $t$-test was used to compare pre- and post-treatment values. The unpaired $t$-test was used to compare means between independent groups. Pearson correlation coefficients were calculated to evaluate the correlations of seminal fluid parameters with $\mathrm{CoQ} 10$ levels and SDF. A false discovery rate (FDR) correction for multiple comparisons was performed using the Benjamini-Hochberg procedure. A $p$-value lower than 0.05 was considered to indicate statistical significance.

\section{Results}

Semen parameters in patients were significantly lower than in the control group. Both CoQ10 and Centrum improved sperm concentration $(p<0.05)$, progressive motility $(p<0.01)$, and total motility $(p<0.05)$; however, sperm motility showed better improvement with Centrum therapy $(p<0.05)$ (Table 1).

Infertile patients showed poorer seminal antioxidant status than the fertile control group (Table 2). Seminal CoQ10 levels were significantly lower in infertile men than in controls and significantly increased following CoQ10 therapy (FDR $p<0.01)$. CoQ10 and Centrum therapy both reduced ROS and DNA fragmentation and improved TAC and CAT activity, but the reduction in ROS (FDR $p<0.01)$ and CAT (FDR $p<0.001$ ) was significantly lower with Centrum therapy and the improvement of TAC (FDR $p<0.01$ ) was significantly higher with CoQ10 treatment (Table 2).

Patients had higher FSH, LH, and prolactin and lower testosterone levels than controls. Centrum therapy resulted in a decrease in serum testosterone in patients $(p<0.05)$ (Figure 1). Correlations were also found between total sperm motility and SDF $(r=-0.51, p=0.002)$ and between total sperm motility and seminal CoQ10 levels $(r=0.42$, $p=0.007$ ) (Table 3).

\section{Discussion}

With an increasing body of evidence linking OS and SDF to male infertility, antioxidant supplementation has been recommended for the treatment of IMI [6]. Both individual and combined antioxidant treatments have been attempted in men with IMI [26]. Recent systematic reviews have examined several studies and reported that antioxidants exert beneficial effects on semen parameters, antioxidant status, and fertility potential in men with IMI [26-28]. However, 
Table 1. Semen parameters in fertile and infertile men before and after administration of CoQ10 and Centrum

\begin{tabular}{|c|c|c|c|c|c|}
\hline Varaible & $\begin{array}{l}\text { Fertile control } \\
\qquad(n=58)\end{array}$ & $\begin{array}{l}\text { Patient before } \\
\text { CoQ10 }(n=65)\end{array}$ & $\begin{array}{c}\text { Patient after } \\
\text { CoQ10 }\end{array}$ & $\begin{array}{l}\text { Patient before } \\
\text { Centrum }\end{array}$ & $\begin{array}{l}\text { Patient after } \\
\text { Centrum }\end{array}$ \\
\hline Age (yr) & $36.4 \pm 15.2$ & $33.6 \pm 14.1$ & & $32.4 \pm 13.6$ & \\
\hline Infertility duration (yr) & & $6.4 \pm 5.2$ & & $7.1 \pm 6.4$ & \\
\hline Volume (mL) & $3.1 \pm 1.6$ & $3.3 \pm 1.8$ & $3.5 \pm 1.6$ & $3.6 \pm 2.0$ & $3.6 \pm 1.7$ \\
\hline Concentration (million/mL) & $50.4 \pm 27.3$ & $8.9 \pm 5.1^{c)}$ & $10.6 \pm .6 .4^{\mathrm{a}), \mathrm{c})}$ & $9.8 \pm 5.7^{c)}$ & $11.8 \pm 7.0^{\mathrm{a}), \mathrm{c})}$ \\
\hline Progressive motility (\%) & $46.1 \pm 9.7$ & $20.8 \pm 8.4^{c)}$ & $25.7 \pm 12.5^{\mathrm{b}), \mathrm{c})}$ & $22.9 \pm 9.3^{c)}$ & $30.2 \pm 13.6^{b), c(, d)}$ \\
\hline Total motility (\%) & $63.5 \pm 12.6$ & $28.8 \pm 8.2^{c)}$ & $35.1 \pm 14.6^{\mathrm{a}), \mathrm{c})}$ & $31.6 \pm 9.2^{c)}$ & $41.4 \pm 16.2^{\mathrm{a}), \mathrm{c}, \mathrm{d})}$ \\
\hline Normal morphology (\%) & $6.3 \pm 2.9$ & $2.7 \pm 2.1^{c)}$ & $3.3 \pm 1.6^{c)}$ & $2.5 \pm 1.3^{\mathrm{c})}$ & $3.7 \pm 1.6^{c)}$ \\
\hline
\end{tabular}

Values are presented as mean \pm standard deviation.

CoQ10, coenzyme Q10.

${ }^{\text {a) }}$ vs. patients before CoQ10, $p<0.05 ;{ }^{\text {b) }}$ vs. patients before CoQ10, $p<0.01 ;{ }^{c}$ vs. fertile control group, $p<0.001 ;{ }^{\text {d) }}$ vs. patients after CoQ10, $p<0.05$.

Table 2. Seminal plasma CoQ10, oxidative stress markers, and sperm DNA fragmentation levels in fertile and infertile men before and after administration of CoQ10 and Centrum

\begin{tabular}{|c|c|c|c|c|c|}
\hline Variable & Fertile control & $\begin{array}{l}\text { Patient before } \\
\text { CoQ10 }\end{array}$ & $\begin{array}{l}\text { Patient after } \\
\text { CoQ10 }\end{array}$ & $\begin{array}{l}\text { Patient before } \\
\text { Centrum }\end{array}$ & $\begin{array}{l}\text { Patient after } \\
\text { Centrum }\end{array}$ \\
\hline CoQ10 level (ng/mL) & $56.2 \pm 38.5$ & $41.6 \pm 29.8^{\mathrm{d})}$ & $76.9 \pm 26.3^{\mathrm{b}), \mathrm{e})}$ & $38.9 \pm 27.6^{d)}$ & $40.2 \pm 28.1^{\mathrm{d}), 9)}$ \\
\hline $\mathrm{ROS}\left(\times 10^{4} \mathrm{RLU} / \mathrm{min} / 20\right.$ million spermatozoa) & $0.07 \pm 0.03$ & $3.52 \pm 1.29^{\mathrm{e})}$ & $2.68 \pm 1.31^{(\mathrm{c}), e)}$ & $2.8 \pm 0.96^{\mathrm{e})}$ & $2.08 \pm 1.04^{(\mathrm{c}, e), f]}$ \\
\hline $\mathrm{TAC}(\mathrm{mmol} / \mathrm{L})$ & $1.12 \pm 0.21$ & $0.73 \pm 0.36^{\mathrm{d})}$ & $0.92 \pm 0.4^{\mathrm{b}), \mathrm{d})}$ & $0.56 \pm 0.24^{d)}$ & $0.73 \pm 0.3^{(\mathrm{c}, \mathrm{d}), \mathrm{f})}$ \\
\hline Catalase $(\mathrm{U} / \mathrm{mL})$ & $12.45 \pm 2.49$ & $8.42 \pm 2.21^{\mathrm{e})}$ & $9.8 \pm 2.06^{b), e)}$ & $6.72 \pm 1.75^{\mathrm{e})}$ & $7.8 \pm 1.4^{b, e), g)}$ \\
\hline Sperm DNA fragmentation (\%) & $13.2 \pm 3.8$ & $35.2 \pm 6.4^{\mathrm{e})}$ & $32.1 \pm 7.9^{\mathrm{a}), \mathrm{e}}$ & $28.3 \pm 5.1^{\mathrm{e})}$ & $25.7 \pm 4.1^{\mathrm{b}), \mathrm{e}, \mathrm{g})}$ \\
\hline
\end{tabular}

CoQ10, coenzyme Q10; ROS, reactive oxygen species; TAC, total antioxidant capacity; FDR, false discovery rate.

${ }^{\text {a) }}$ vs. patients before CoQ10, FDR $p<0.05 ;{ }^{\text {b) }}$ vs. patients before CoQ10, FDR $p<0.01$; ${ }^{\text {c) }}$ vs. patients before CoQ10, FDR $p<0.001$; ${ }^{\text {d) }}$ vs. fertile control group, FDR $p<0.01$; ${ }^{\text {e) }}$ vs. fertile control group, FDR $p<0.001$; ${ }^{\text {f) }}$ vs. patients after CoQ10, FDR $p<0.01 ;{ }^{\text {g) }}$ vs. patients after CoQ10, FDR $p<0.001$.

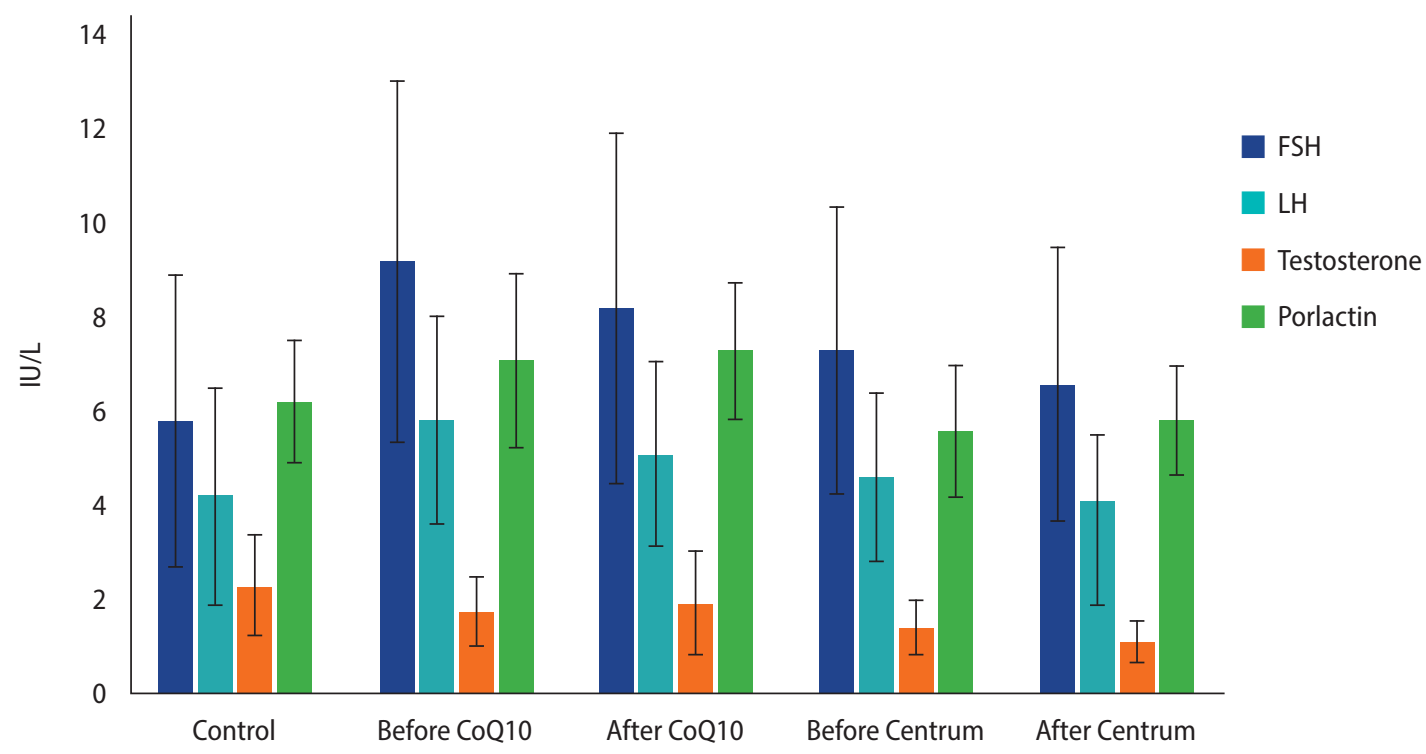

Figure 1. Hormones in fertile and infertile men before and after administration of coenzyme Q10 (CoQ10) and Centrum. FSH, folliclestimulating hormone; LH, luteinizing hormone. 
Table 3. Correlations between SDF, CoQ10 levels, and semen parameters in infertile subjects after CoQ10 treatment

\begin{tabular}{lccc}
\hline \multirow{2}{*}{ Variable } & \multicolumn{3}{c}{$r(p$-value $)$} \\
\cline { 2 - 4 } & Concentration & Total motility & Normal morphology \\
\hline SDF & $-0.07(0.34)$ & $-0.51(0.002)$ & $-0.14(0.24)$ \\
CoQ10 & $0.23(0.16)$ & $0.42(0.007)$ & $0.11(0.26)$
\end{tabular}

$r$ : Pearson correlation coefficient.

SDF, sperm DNA fragmentation; CoQ10, coenzyme Q10.

there is a lack of consensus on the type, dosing, target group, and the use of individual or combined antioxidants in IMI [27]. To our knowledge, this study is the first to compare the effects of CoQ10 and Centrum on semen parameters, seminal antioxidant capacity, and SDF in men with idiopathic OA.

In our study, both CoQ10 and Centrum improved sperm concentration, progressive motility, and total motility; however, sperm motility showed better improvement with Centrum therapy. A randomized, double-blind, placebo-controlled trial of 212 men with idiopathic oligoasthenoteratospermia (OAT) who received CoQ10 (300 $\mathrm{mg} /$ day) for 26 weeks reported improvements in sperm concentration and motility post-therapy [29]. Balercia et al. [21,30], in two studies of 82 men with idiopathic asthenospermia treated with CoQ10 (200 mg/day) for 6 months, also confirmed higher sperm progressive and total motility and an increase in seminal CoQ10 level after treatment. Furthermore, our recent systematic review and another systematic review including three randomized clinical trials in infertile men who received CoQ10 therapy confirmed improvements in sperm concentration and motility in these men, although there was no increment in pregnancy rates [31,32]. Another study, however, reported no improvement in semen parameters in men with idiopathic OAT following CoQ10 therapy [33].

We could not find studies on the effects of Centrum on semen parameters or antioxidant status or in comparison with the effects of CoQ10 therapy. However, many studies have tried combinations of multiple antioxidants in men with IMI and reported improvement in 1 or more semen parameters $[34,35]$ and in the pregnancy rate [36]. One study reported no improvement in semen parameters or pregnancy rates after combined antioxidant treatment [37]. As expected, infertile men had lower semen parameters than fertile controls. The improvement in semen parameters observed in our study could be attributed to the antioxidant properties of $\mathrm{CoQ} 10$ and Centrum multivitamins, which counteract OS and its detrimental effects on sperm in men with idiopathic OA [6]. Nevertheless, a comparison with the results of the aforementioned studies is challenging due to the heterogeneity of design, antioxidants, doses, and treatment duration.

Seminal CoQ10 levels were significantly lower in infertile men than in controls and significantly increased following CoQ10 therapy. CoQ10 and Centrum therapy both reduced ROS and DNA fragmentation and improved TAC and CAT activity, but the reduction in ROS and SDF was significantly lower with Centrum therapy and the improvement of TAC and CAT was significantly higher with CoQ10 treatment. Our results are consistent with previous studies that demonstrated lower seminal antioxidant capacity [12] and higher SDF in infertile men than in controls [38]. Our previous studies on men with idiopathic OA and OAT treated with CoQ10 (200 mg/day) for 3 months demonstrated improvements in semen parameters, ROS, TAC, CAT, and GPx, as well as a reduction in SDF following CoQ10 therapy $[13,18,39,40]$. Our findings are consistent with other studies showing that CoQ10 therapy resulted in improvement in antioxidant capacity and reduced SDF levels in infertile men [13,41]. Our study also demonstrated a correlation between seminal CoQ10 and SDF levels and sperm total motility. This finding aligns with our previous studies, which have also shown correlations between CoQ10 levels, SDF, and sperm motility [13,18]. Other studies have also reported similar correlations in men with IMI [42,43]. We could not find studies on the effect of Centrum on SDF, but several studies have explored the impact of different combinations of antioxidants in IMI and reported reductions in SDF levels $[44,19]$. Other studies, however, reported no alterations in SDF levels after antioxidant treatment $[45,46]$. CoQ10 treatment in our study resulted in decreased FSH and LH levels, but this change was not statistically significant, and there was a reduction in serum testosterone with Centrum treatment. The reduction in serum testosterone after Centrum therapy was an unexpected finding, which might have been due to the direct effects of 1 or more of the 26 antioxidants in Centrum on seminiferous tubules. This possibility requires further investigation. A previous study of men with idiopathic OAT treated with CoQ10 (300 mg/day) reported reductions in $\mathrm{FSH}$, $\mathrm{LH}$, and inhibin levels [29].

The improvement in antioxidant capacity and reduction in SDF level detected in our study after CoQ10 and Centrum therapy could be due to lower antioxidant capacity in infertile men and the antioxidant properties of CoQ10 and Centrum multivitamins [12]. These properties may counteract $O S$, increase seminal antioxidant defense, and reduce OS-induced SDF and therefore may enhance fertility potential in men with idiopathic OA [14]. The greater improvement after Centrum therapy could have been due to the synergistic antioxidant action of the 26 combined antioxidants. Comparisons of the studies discussed above are also limited by the heterogeneity of studies' designs and the different combinations of antioxidants used. The correlations between semen parameters and antioxidant capacity and SDF may establish the foundation for the use of oral antioxidants, including CoQ10, in the treatment of infertile men with IMI and idiopathic $\mathrm{OA}$ to enhance their pregnancy outcomes [7]. Fur- 
thermore, these measures could also be used as diagnostic biomarkers for male fertility and pregnancy outcomes. The limitations of our study include the small sample size and the lack of long-term follow-up, which meant that we could not report the pregnancy rate for the participants. Therefore, further large-scale studies are warranted to consolidate the findings of this study.

Both CoQ10 (200 mg/day) and Centrum (1 tablet/day) treatment for 3 months were effective in improving semen parameters, antioxidant capacity, and reducing SDF, but the improvement was greater with Centrum than with CoQ10. Therefore, Centrum combined antioxidants may provide more effective results than individual antioxidants such as CoQ10 in the treatment of infertile men with idiopathic OA due to the potential synergistic antioxidant action of the combined antioxidants. Furthermore, semen parameters, seminal antioxidant capacity, and SDF could be used as diagnostic biomarkers in men with IMI.

\section{Conflict of interest}

No potential conflict of interest relevant to this article was reported.

\section{Acknowledgments}

The authors would like to express their sincere gratitude to all patients who participated in this study for their valuable help in accomplishing this work.

\section{ORCID}

$\begin{array}{ll}\text { Ahmed T Alahmar } & \text { https://orcid.org/0000-0003-2100-5807 } \\ \text { Rajender Singh } & \text { https://orcid.org/0000-0002-4592-6566 }\end{array}$

\section{Author contributions}

Conceptualization: ATA. Data curation: ATA. Formal analysis: ATA. Methodology: ATA. Project administration: ATA. Visualization: ATA. Writing-original draft: ATA. Writing-review \& editing: RS.

\section{References}

1. Layali I, Tahmasbpour E, Joulaei M, Jorsaraei SG, Farzanegi P. Total antioxidant capacity and lipid peroxidation in semen of patient with hyperviscosity. Cell J 2015;16:554-9.

2. Mascarenhas MN, Flaxman SR, Boerma T, Vanderpoel S, Stevens GA. National, regional, and global trends in infertility prevalence since 1990: a systematic analysis of 277 health surveys. PLoS Med 2012;9:e1001356.

3. Thoma ME, McLain AC, Louis JF, King RB, Trumble AC, Sundaram R, et al. Prevalence of infertility in the United States as estimated by the current duration approach and a traditional constructed approach. Fertil Steril 2013;99:1324-31.

4. Kupis L, Dobroński PA, Radziszewski P. Varicocele as a source of male infertility: current treatment techniques. Cent European J Urol 2015;68:365-70.

5. Chehab M, Madala A, Trussell JC. On-label and off-label drugs used in the treatment of male infertility. Fertil Steril 2015;103: 595-604.

6. Agarwal A, Parekh N, Panner Selvam MK, Henkel R, Shah R, Homa ST, et al. Male Oxidative Stress Infertility (MOSI): proposed terminology and clinical practice guidelines for management of idiopathic male infertility. World J Mens Health 2019;37:296-312.

7. Arafa M, Agarwal A, Majzoub A, Selvam MK, Baskaran S, Henkel R, et al. Efficacy of antioxidant supplementation on conventional and advanced sperm function tests in patients with idiopathic male infertility. Antioxidants (Basel) 2020;9:219.

8. Leaver RB. Male infertility: an overview of causes and treatment options. Br J Nurs 2016;25:S35-40.

9. Skoracka K, Eder P, Lykowska-Szuber L, Dobrowolska A, Krela-Kazmierczak I. Diet and nutritional factors in male (in)fertility: underestimated factors. J Clin Med 2020;9:1400.

10. Bisht S, Faiq M, Tolahunase M, Dada R. Oxidative stress and male infertility. Nat Rev Urol 2017;14:470-85.

11. Agarwal A, Tvrda E, Sharma R. Relationship amongst teratozoospermia, seminal oxidative stress and male infertility. Reprod Biol Endocrinol 2014;12:45.

12. Huang C, Cao X, Pang D, Li C, Luo Q, Zou Y, et al. Is male infertility associated with increased oxidative stress in seminal plasma?: a-meta analysis. Oncotarget 2018;9:24494-513.

13. Alahmar AT, Sengupta P, Dutta S, Calogero AE. Coenzyme Q10, oxidative stress markers, and sperm DNA damage in men with idiopathic oligoasthenoteratospermia. Clin Exp Reprod Med 2021;48: $150-5$.

14. Nguyen-Powanda P, Robaire B. Oxidative stress and reproductive function in the aging male. Biology (Basel) 2020;9:282.

15. Balercia G, Mancini A, Paggi F, Tiano L, Pontecorvi A, Boscaro M, et al. Coenzyme Q10 and male infertility. J Endocrinol Invest 2009; 32:626-32.

16. Alahmar AT. Role of oxidative stress in male infertility: an updated review. J Hum Reprod Sci 2019;12:4-18.

17. Majzoub A, Agarwal A, Esteves SC. Antioxidants for elevated sperm DNA fragmentation: a mini review. Transl Androl Urol 2017;6(Suppl 4):S649-53.

18. Alahmar AT, Calogero AE, Sengupta P, Dutta S. Coenzyme Q10 im- 
proves sperm parameters, oxidative stress markers and sperm DNA fragmentation in infertile patients with idiopathic oligoasthenozoospermia. World J Mens Health 2021;39:346-51.

19. Gual-Frau J, Abad C, Amengual MJ, Hannaoui N, Checa MA, Ribas-Maynou J, et al. Oral antioxidant treatment partly improves integrity of human sperm DNA in infertile grade I varicocele patients. Hum Fertil (Camb) 2015;18:225-9.

20. Ahmadi S, Bashiri R, Ghadiri-Anari A, Nadjarzadeh A. Antioxidant supplements and semen parameters: an evidence based review. Int J Reprod Biomed 2016;14:729-36.

21. Balercia G, Buldreghini E, Vignini A, Tiano L, Paggi F, Amoroso S, et al. Coenzyme Q10 treatment in infertile men with idiopathic asthenozoospermia: a placebo-controlled, double-blind randomized trial. Fertil Steril 2009;91:1785-92.

22. World Health Organization. WHO laboratory manual for the examination and processing of human semen. 5th ed. Geneva: World Health Organization; 2010.

23. Li K, Shi Y, Chen S, Li W, Shang X, Huang Y. Determination of coenzyme Q10 in human seminal plasma by high-performance liquid chromatography and its clinical application. Biomed Chromatogr 2006;20:1082-6.

24. Venkatesh S, Shamsi MB, Dudeja S, Kumar R, Dada R. Reactive oxygen species measurement in neat and washed semen: comparative analysis and its significance in male infertility assessment. Arch Gynecol Obstet 2011;283:121-6.

25. Zaazaa A, Adel A, Fahmy I, Elkhiat Y, Awaad AA, Mostafa T. Effect of varicocelectomy and/or mast cells stabilizer on sperm DNA fragmentation in infertile patients with varicocele. Andrology 2018;6: 146-50.

26. Alahmar AT. The effects of oral antioxidants on the semen of men with idiopathic oligoasthenoteratozoospermia. Clin Exp Reprod Med 2018;45:57-66.

27. Majzoub A, Agarwal A. Systematic review of antioxidant types and doses in male infertility: benefits on semen parameters, advanced sperm function, assisted reproduction and live-birth rate. Arab J Urol 2018;16:113-24.

28. Agarwal A, Leisegang K, Majzoub A, Henkel R, Finelli R, Panner Selvam MK, et al. Utility of antioxidants in the treatment of male infertility: clinical guidelines based on a systematic review and analysis of evidence. World J Mens Health 2021;39:233-90.

29. Safarinejad MR. Efficacy of coenzyme Q10 on semen parameters, sperm function and reproductive hormones in infertile men. J Urol 2009;182:237-48.

30. Balercia G, Mosca F, Mantero F, Boscaro M, Mancini A, Ricciardo-Lamonica G, et al. Coenzyme Q(10) supplementation in infertile men with idiopathic asthenozoospermia: an open, uncontrolled pilot study. Fertil Steril 2004;81:93-8.
31. Vishvkarma R, Alahmar AT, Gupta G, Rajender S. Coenzyme Q10 effect on semen parameters: profound or meagre? Andrologia 2020;52:e13570.

32. Lafuente R, Gonzalez-Comadran M, Sola I, Lopez G, Brassesco M, Carreras R, et al. Coenzyme Q10 and male infertility: a meta-analysis. J Assist Reprod Genet 2013;30:1147-56.

33. Nadjarzadeh A, Sadeghi MR, Amirjannati N, Vafa MR, Motevalian SA, Gohari MR, et al. Coenzyme Q10 improves seminal oxidative defense but does not affect on semen parameters in idiopathic oligoasthenoteratozoospermia: a randomized double-blind, placebo controlled trial. J Endocrinol Invest 2011;34:e224-8.

34. Busetto GM, Koverech A, Messano M, Antonini G, De Berardinis E, Gentile V. Prospective open-label study on the efficacy and tolerability of a combination of nutritional supplements in primary infertile patients with idiopathic astenoteratozoospermia. Arch Ital Urol Androl 2012;84:137-40.

35. Piomboni P, Gambera L, Serafini F, Campanella G, Morgante G, De Leo V. Sperm quality improvement after natural anti-oxidant treatment of asthenoteratospermic men with leukocytospermia. Asian J Androl 2008;10:201-6.

36. Kobori Y, Ota S, Sato R, Yagi H, Soh S, Arai G, et al. Antioxidant cosupplementation therapy with vitamin $\mathrm{C}$, vitamin $\mathrm{E}$, and coenzyme Q10 in patients with oligoasthenozoospermia. Arch Ital Urol Androl 2014;86:1-4.

37. Tremellen K, Miari G, Froiland D, Thompson J. A randomised control trial examining the effect of an antioxidant (Menevit) on pregnancy outcome during IVF-ICSI treatment. Aust N Z J Obstet Gynaecol 2007;47:216-21.

38. Agarwal A, Majzoub A, Baskaran S, Panner Selvam MK, Cho CL, Henkel R, et al. Sperm DNA fragmentation: a new guideline for clinicians. World J Mens Health 2020;38:412-71.

39. Alahmar AT. The impact of two doses of coenzyme Q10 on semen parameters and antioxidant status in men with idiopathic oligoasthenoteratozoospermia. Clin Exp Reprod Med 2019;46:1128.

40. Alahmar AT, Sengupta P. Impact of coenzyme Q10 and selenium on seminal fluid parameters and antioxidant status in men with idiopathic infertility. Biol Trace Elem Res 2021;199:1246-52.

41. Abad C, Amengual MJ, Gosalvez J, Coward K, Hannaoui N, Benet J, et al. Effects of oral antioxidant treatment upon the dynamics of human sperm DNA fragmentation and subpopulations of sperm with highly degraded DNA. Andrologia 2013;45:211-6.

42. Nadjarzadeh A, Shidfar F, Amirjannati N, Vafa MR, Motevalian SA, Gohari MR, et al. Effect of Coenzyme Q10 supplementation on antioxidant enzymes activity and oxidative stress of seminal plasma: a double-blind randomised clinical trial. Andrologia 2014;46: 177-83. 
43. Boushaba S, Belaaloui G. Sperm DNA fragmentation and standard semen parameters in algerian infertile male partners. World J Mens Health 2015:33:1-7.

44. Salehi P, Zahra Shahrokhi S, Kamran T, Ajami A, Taghiyar S, Reza Deemeh M. Effect of antioxidant therapy on the sperm DNA integrity improvement; a longitudinal cohort study. Int J Reprod Biomed 2019;17:99-106.

45. Stenqvist A, Oleszczuk K, Leijonhufvud I, Giwercman A. Impact of antioxidant treatment on DNA fragmentation index: a double-blind placebo-controlled randomized trial. Andrology 2018;6: 811-6.

46. Steiner A, Hansen K, Diamond M, Coutifaris C, Cedars M, Legro R, et al. Antioxidants in the treatment of male factor infertility: results from the double blind, multi-center, randomized controlled Males, Antioxidants, and Infertility (MOXI) trial. Hum Reprod 2018:33:i30. 